\section{Necesidad de creación, por ley, de un programa de orientación, preparación y capacitación del trabajador que jubilará}

\author{
Need to create by law of, a program of guidance, preparation \\ and training of the worker to retire
}

\begin{abstract}
RESUMEN
La jubilación representa, para el trabajador, la satisfacción de haber cumplido con su misión en la vida. La edad de jubilación del trabajador en la ley tiene componentes costumbristas, históricos, biológicos y de carácter universal. La ley no le consulta al trabajador si se siente en capacidad de continuar en su trabajo aun cuando esta ley le diga que debe retirarse. La ley no contempla el tipo de labor que realiza el trabajador, como es el caso del desempeño en actividades intelectuales, innovadoras, legislativas, organizativas, y actividades en las que el trabajador se desempeña con rendimientos de alta productividad; a pesar de encontrarse, según ley, en edad de retiro. Estas y otras situaciones exigen la existencia de un programa de orientación, preparación y capacitación al trabajador. El mayor énfasis que se debe poner, en este tipo de propuesta, está en preparación psicológica y capacitación al trabajador para su desempeño independiente; como lo es en países desarrollados con alta Responsabilidad Social. La inexistencia de este programa en nuestro país constituye un enorme vacío social.

Palabras claves: Jubilación; ingresos suplementarios; emprendimiento en los ancianos.
\end{abstract}

\begin{abstract}
Retirement represents for the worker the satisfaction of having fulfilled his mission in life. The retirement age of the worker in the law, has costumbristas, historical, biological and universal components. The law does not consult the worker if he feels able to continue in his work even when the law tells him to retire, the law does not contemplate the type of work that the worker performs, as is the case of performance in intellectual activities, Innovative, legislative, organizational, and activities in which the worker performs with high productivity yields despite being according to law at retirement age. These and other situations demand the existence of a program of orientation, preparation and training to the worker. The greatest emphasis that must be placed on this type of proposal is in psychological preparation and training the worker for their independent performance as it is in developed countries with high social responsibility, the inexistence of this program in our country constitutes a huge social vacuum.

Keywords: Retirement; supplementary income; entrepreneurship in the elderly.
\end{abstract}

\section{Juan Manuel Barreda Guerra*}

barredaguerra@yahoo.es

Universidad Nacional Mayor de San Marcos, Facultad de Ciencias Administrativas

+ Docente Asociado de la Universidad Nacional Mayor de San Marcos

(C) Los autores. Este artículo es publicado por Gestión en el Tercer Milenio de la Facultad de Ciencias Administrativas de la Universidad Nacional Mayor de San Marcos. Este es un artículo de acceso abierto, distribuido bajo los términos de la licencia Creative Commons Atribucion - No Comercia_Compartir Igual 4.0 Internacional. (http://creativecommons.org/licenses/by-nc-sa/4.0/) que permite el uso no comercial, distribución y reproducción en cualquier medio, siempre que la obra original sea debidamente citada. 


\section{INTRODUCCIÓN}

La jubilación representa, de cierto modo para un ciudadano trabajador la satisfacción de haber cumplido con su misión en la vida. De igual forma esto conlleva a una satisfacción personal, familiar y social, en el digno rol y estatus de su desempeño en la vida.

En este sentido, la ley en su actuar impositivo y drástico, no consulta para nada al trabajador sobre su voluntad o deseo de retirarse. Al actuar de este modo, la ley yerra y colisiona contra un derecho humano de palpitante vigencia, al descontinuar al trabajador en su derecho laboral en función de su edad; lo cual constituye discriminación, toda vez que el trabajador se encuentre las perfectas condiciones psíquicas y físicas y, aun así, sea sometido a su separación del contexto productivo nacional. Como resultado, esto trae consigo el engrosamiento de un gran grupo las filas de un grupo vulnerable como es la masa crítica jubilada en el Perú. Se trata pues de hombres y mujeres mayores que dieron todo a su sociedad para el desarrollo económico y bienestar de su país y que, luego por ley, fueron condenados a la pobreza y engrosar las filas del grupo vulnerable de los jubilados.

Como quiera que fuere y se aplique la ley de retiro o jubilación en nuestro país, sus beneficios y bondades, por lo general, tardan años en su aplicabilidad y muchos no alcanzan a gozar de sus "beneficios"; empero, lo que sí sabemos por experiencia vivencial y práctica como ciudadano y sociedad, es que en nuestro país se dan leyes de tipo y corte casi celestial, maravillosas, esplendidas, tal vez únicas en el planeta; empero, tan solo en el papel. Vale decir, que las leyes no se cumplen en razón de que para su aplicabilidad requiere de lo fundamental como es su presupuesto de operativo y, en nuestro país, se acostumbra dar leyes. Luego, es necesario esperar sus reglamentación y posteriormente su implementación presupuestal, la habilitación de infraestructuras, equipos y personal para su aplicación. Esto, por lo general, toma su tiempo, (comúnmente una década); mientras tanto, los ciudadanos siguen esperando las bondades de la ley, el tiempo pasa y los ciudadanos no son acogidos en la debida forma que la señala.
Mientras tanto, la vida y sus carencias siguen afectando esta población objetivo. Este es el caso de los jubilados en nuestro país.

\section{PLANTEAMIENTO DEL PROBLEMA PROBLEMA}

¿La Ley de jubilación en Perú obliga al ciudadano trabajador a salir del sistema productivo y perder un alto porcentaje de su poder adquisitivo que lo empobrece y convierte en ciudadano vulnerable, aun cuando pueda encontrarse este en buenas condiciones psíquicas y físicas para su desempeño eficiente y productivo?

\section{OBJETIVO GENERAL}

Medir la predisposición en los potenciales cercanos a la jubilación de capacitarse para emprender, con sus familias, un negocio que le permita obtener mejores ingresos, en comparación con la devolución que pueda recibir al jubilarse en una AFP.

\section{OBJETVOS ESPECÍFICOS}

Objetivo Específico 1: Medir la predisposición a la asociatividad en el trabajador cercano a su jubilación con fines productivos.

Objetivo Específico 2: Medir en el empresariado de la Gran Lima, los reparos en demandar productos y/o servicios de empresas conformadas por personas jubiladas.

Objetivo Específico 3: Medir en las amas de casa de la Gran Lima, sus reparos en adquirir productos y/o servicios ofertados por empresas conformadas y dirigidas por jubilados.

\section{JUSTIFICACIÓN}

Nuestro país no cuenta con una ley de preparación psíquica y formativa para el trabajador que se encuentra próximo a su salida del mercado laboral, por haber llegado al límite de edad considerada productiva, vale decir, 65 años para el varón y 60 para las damas. Como se ha dicho en la investigación, este límite en la edad contiene factores y aspectos de tipo costumbrista, biológicos, culturales, tecnológicos, sociales y económicos ancestrales y no considera; para nada, el sentir y la opinión del trabajador desde su punto de vista psíquico 
y físico y, simplemente debe y tiene que cumplir la ley ${ }^{1}$; aun cuando se pueda encontrarse en un nivel de desempeño altamente productivo.

Países como Alemania, Bélgica, Holanda, Suiza, Japón cuentan con normatividad laboral que obligan a la empresa y trabajador a prepararlo psicológicamente para su salida del mercado laboral con la debida anticipación. De esta manera se evita un shock emocional en la persona, depresión, sentimientos de inutilidad, soledad y vejez. Se le prepara al trabajador con dos a tres años de anticipación a su jubilación en aspectos psíquicos y, sobre todo, en relación a las actividades de recreación, distracción, viajes, hobbies, administración de su tiempo libre, construcción de ideas, proyectos, participaciones sociales; entre todo un bagaje de opciones y posibilidades en las que el jubilado podrá participar para disfrutar y optimizar su tiempo, sin dramatizar su retiro laboral. Téngase en consideración que, en estos países, el jubilado percibe una pensión digna y decente por su jubilación que le permite verdaderamente gozar del júbilo que debe sentir la persona que ha cumplido con su misión social y con él mismo, con su familia y sociedad. Si a estos beneficios le añadidos el incremento de la esperanza de vida en estos países, podremos apreciar que es mayor el tiempo que tienen las personas adultas mayores de la tercera edad para gozar de su jubilación, con relación a nuestros jubilados peruanos.

En nuestro país, no contamos con programas de preparación de esta naturaleza para la salida del mercado laboral; por el contrario, el trabajador que se jubila ve caer su poder adquisitivo en más del $60 \%$; así como las posibilidades de empleo en el mercado laboral para el jubilado que son nulas. En razón de esta circunstancia, se requiere la creación, mediante Ley, de un programa de capacitación y preparación para jubilados del sector público y privado; que permita la formación de la persona jubilada a fin que esta sea capaz de desempeñarse independientemente ${ }^{2}$ en la

\footnotetext{
${ }^{1}$ Acta bioeth. v.7 n.1 Santiago 2001- El mundo moderno: el viejo frente a la burocracia http://dx.doi.org/10.4067/S1726569X2001000100008-

${ }^{2}$ Andrew Shepherd (2004) Una mirada a la protección social desde los derechos humanos y otros contextos internacionales. Publicación de las Naciones Unidas CEPAL pág. - 7. ISSN impreso 1564-4162 ISSN electrónico 1680-8983.
}

creación de riqueza y generación propia de mayores ingresos para él y su familia, logrando, de este modo, su autodesarrollo y suficiencia para vivir dignamente. De igual forma el disfrute de una vida tranquila y placentera, realizando lo que realmente siempre deseó hacer y disfrutar el trabajador que se retira en cumplimiento de la ley.

\section{Ingresos}

Diversidad de instituciones internacionales, profesionales y universidades en distintas latitudes han demostrado, hasta la saciedad, que los ingresos promedios de la persona que decide jubilarse a su turno o anticipadamente, sea varón o dama, entrarán en caída libre a un rango que equivaldrá entre el 35 al 40\% de su ingreso promedio. La caída de esta es muy considerable y solo se acepta cuando la persona es obligada por ley o razones de salud que no le permiten la continuidad en su actividad laboral de costumbre. De no existir estos factores coercitivos para el retiro, un alto porcentaje de la población laboral mayor de 65 años continuaría sin ningún problema en su quehacer laboral; en razón de que con ingresos tan bajos como los que ofrece la jubilación, no se puede mantener lo logrado. Por lo tanto, la jubilación en nuestro país, más que prevención social, es una condena al empobrecimiento de la persona.

\section{Jubilación, falso concepto de disminución de la productividad}

En la actualidad, la combinación del trabajado del hombre con la tecnología es bastante amplia; su mayor participación está en el conocimiento $^{3}$, y no en la fuerza física. El binomio hombre máquina exige que los dos mantengan una información y comunicación acorde al trabajo que realizarán ${ }^{4}$ ambos, y que con seguridad, serán coordinados e integrados a otros procesos, sean estos de producción, ventas, logísticas, finanzas, entre otros.

\footnotetext{
${ }^{3}$ Andrew Shepherd (2004) Una mirada a la protección social desde los derechos humanos y otros contextos internacionales. Publicación de las Naciones Unidas ISSN impreso 1564-4162 ISSN electrónico 1680-8983.

${ }^{4}$ Barraza de Anda M. La Productividad Social en las universidades Públicas en México- XI Congreso Internacional sobre la Reforma del Estado y la Administración Pública.
} 
En nuestro país, para la atención de las necesidades poblacionales internas se necesita emplear, de modo abundante, nuestra mano de obra tecnificada, eficiente y capaz. No debemos caer en el error de desplazar al trabajador, esto producirá desempleo; el desempleo genera pobreza; la pobreza no permite que se desarrollen mercado internos; la falta de mercados internos mata las empresas; la muerte de las empresas genera pérdidas para los inversionistas; las pérdidas de los empresarios no le permiten al estado recaudar impuestos; la falta de recaudación de impuestos impide al Estado solventar presupuestos; no contar con tener dinero en el Estado genera crisis y caos económico que afecta a todos los peruanos. En la medida que podamos ir generando cada vez más empleo para todos, mejorarán los niveles de vida de la población. He ahí la importancia de generar, en nuestro país, empleo adecuado y sostenible. La productividad viene entonces en el adecuado uso y rendimiento de los factores de producción y el máximo rendimiento de los mismos en el resultado logrado.

Bajo los conceptos vertidos líneas arriba, se entiende que el jubilado no pondrá un negocio, a su edad para cargar pesos, caminar y moverse durante todo el día. Su experiencia y conocimiento lo capacitan para planificar, dirigir y controlar su pequeño negocio. Habrá actividades que él sabrá delegar a trabajadores más jóvenes de la empresa. Para concluir, debe quedar en claro que, la productividad, como aspecto relevante para la competitividad empresarial, no le exigirá al jubilado en su empresa que maximice su trabajo físico ${ }^{5}$; sino, la gestión eficiente de su empresa, lo cual si podrá logarlo con capacitación y preparación.

\section{Perfil de un ciudadano jubilado promedio en nuestro país.}

- Sea varón o dama que pertenece a la población del adulto mayor de la tercera edad.

- Persona con estado de ánimo, probablemente un poco cansado, pero al mismo tiempo gran deseo de hacer algunas cosas que no pudo realizar

${ }^{5}$ Castells, M. (2000). “Era de la información, la economía, sociedad y cultura: la sociedad red". Volumen 1. Siglo veintiuno Editores. 580 páginas. en su edad productiva (Viajar, leer, tener hobbies, estar con la familia, divertirse un poco, visitar familiares en el interior del país o en el extranjero, entre muchas otras distracciones más)

- Según las estadísticas; su proyección de vida se encuentra en un rango de 10 a 15 años de supervivencia luego de su jubilación.

- Sus ingresos económicos han descendido significativamente en relación a lo que recibía en sus últimos sueldos en su edad productiva.

- Probablemente; la persona se encuentre con algunas afecciones propia de su edad o su conyugue; por tanto, se requiere destinar mayor atención, gasto y cuidado médico.

- En su estado anímico se siente en capacidad de poder seguir trabajando para mejorar sus ingresos.

- No halla o no tiene claro cómo autogenerar mayores ingresos.

- Se encuentra proclive a prepararse estudiando o aprendiendo cómo manejar un pequeño negocio.

- Posee amplia experiencia laboral en su campo, mas no en cómo funcionan y operan los negocios.

- Son personas que no recibirán ningún tipo de apoyo por el Estado en lo concerniente a formar su pequeño negocio, a pesar que trabajaron $y$ formaron familias por el bien y el desarrollo económico social de su país.

- Los jubilados son personas adultas mayores que viven en familia, siendo posteriormente esta parte del sostén del jubilado.

- Los jubilados son personas que se han esforzado y trabajando abrigando el deseo de envejecer y vivir en su propia casa, vale decir, que son personas que no desean vivir su vejez en un albergue ni mucho menos de la caridad. 
- Dentro de la población de jubilados existen los denominados jubilados jóvenes. Este término se refiere a los que se jubilaron antes de la edad límite (Lehr y Pholman ${ }^{6}, 2000$ ). Este término se genera dentro de la realidad española en la que el promedio de edad de los jubilados alcanza los 59 años y no la edad de los 65 años.

\section{HIPÓTESIS GENERAL}

Hipótesis General: Los trabajadores, que se encuentran en condiciones de jubilarse, no se sienten en condiciones psicológicas ni físicas de capacitarse para emprender un negocio con su familia.

\section{HIPÓTESIS ESPECIFICAS}

Hipótesis Específica 1: Las empresas, formadas por trabajadores jubilados asociados, no serían bien vistas por los empresarios de la Gran Lima.

Hipótesis Específica 2: Las amas de casa de la Gran Lima no tendrían confianza a los productos y/o servicios ofrecidos por empresas asociativas por jubilados.

\section{MARCO TEORICO}

El retiro laboral es la denominación que se da en algunos países a la jubilación del trabajador. Este se encuentra sujeto a ciertas condiciones legales laborales, mediante las cuales el varón (que ha cumplido 65 años de edad en nuestro país) y la mujer, que ha cumplido sus 60 años, pueden acogerse a la condición social de jubilados. Estos límites de tiempo están basados en parámetros que consideran criterios antiguos ${ }^{7}$ como, por ejemplo, los 70 años como límite máximo de sobrevivencia; cuando en realidad con el avance de la ciencia médica hoy es en muchos países hasta los 78 años y, dependiendo también de cómo se siente en su salud psíquica y física por quien ha llegado a dicha edad. Para ello, citaremos algunos estudios realizados por la OMS en ocasión de celebrar el día mundial de la Salud. Realizó una encuesta a 490 personas

\footnotetext{
${ }^{6}$ Lehr y Pholman, 2000 Participación de las personas mayores en el mercado de trabajo y en la sociedad. Revista Española de Geriatría y Gerontología, 39, 180-84.

${ }^{7}$ Centro de Innovación de la Universidad de Chile (2014-2) Los emprendimientos por necesidad a los emprendimientos por oportunidad. Denise Díaz.- Cristhian A. Cansino
}

mayores de 65 años a las que se les preguntó ¿A qué edad se puede referir la "tercera edad", "adulto mayor" o "vejez"? El promedio de las respuestas fue a los 68 años, lo que no coincidía con los 60 años que consideraba la OMS.

El estudio del observatorio y la Facultad de Ciencias Médicas de la Universidad de UFASTA encontró, en la población encuestada, otros aspectos referentes al ser "viejo" y esto fue que solo el $24 \%$ de estas personas menores de 71 años se sentían incluidas en la tercera edad; lo cual es significativamente nuevo en la psique de las personas menores de 70 años ya que, aproximadamente un $80 \%$ de ellas consideraban que la tercera edad comenzaba antes de los 70 años.

\section{MÉTODO}

Investigación de tipo aplicada con metodología exploratoria descriptiva y cualicuantitativa.

En lo referente a la metodología exploratoria, se han revisado, libros, artículos, revistas, páginas, web, blogs y publicaciones referentes al tema del emprendedurismo en el adulto mayor; tanto de orden nacional como internacional. Lo que han permitido construir el Marco teórico de la Investigación.

De igual forma, en la aplicación de la metodología cuantitativa se han aplicado muestreo simple no estratificado en amas de casa, adultos mayores jubilados y empresarios*. En este último estrato se usó el método de muestreo por conveniencia. En los tres estratos estudiados se aplicaron instrumentos diseñados bajo modalidad de cuestionario, con preguntas que permitieron medir las variables contenidas en el problema, hipótesis y objetivos. Dichos instrumentos fueron probados mediante uso de pilotos para comprobar la efectividad de las preguntas y sus respuestas, en relación con las variables en estudio. El trabajo de campo de la investigación se realizó en diversos distritos de Lima Metropolitana durante los meses de mayo a julio del 2016.

\section{RESULTADOS}

La investigación permitió demostrar que los jubilados y trabajadores por jubilarse, se sienten altamente motivados para capacitarse $\mathrm{y}$, en condiciones psíquicas y físicas para 
continuar desempeñándose de manera independientemente, para poder producir ingresos constantes para sus familias.

Las empresas, que pudieran estar formadas por jubilados, gozarían de amplio apoyo y respaldo por el mercado de amas de casa y de empresarios que estarían dispuestos a trabajar con ellos.

Sobre la base de ponderación de resultados, la investigación ha encontrado un significativo cambio de actitud y tendencia en el jubilado actual, referente a su continuidad en la actividad laboral ${ }^{8}$ en relación a la actitud de los jubilados de hace dos décadas atrás. Quienes a su edad de retiro consideraban que deberían descansar en virtud a que aceptaban con mayor disponibilidad y, si se quiere podría decirse, la aceptación con beneplácito de su retiro a los sesenta años, siendo hoy a los 65 años de edad y con mayor resistencia por los trabajadores a su retiro por considerar ellos que, aún poseen fuerzas para continuar trabajando por ellos y sus familias. $24 \%$ de los Jubilados, tienen pensado hoy formar sus propias empresas con el fin de continuar en actividad laboral**, en razón de que, son conscientes que el cálculo de las devoluciones de sus ahorros que le hará el Sistema Privado de Pensiones son precarias para su supervivencia y la de su esposa.

En cuanto a los trabajadores próximos a su retiro laboral, la tendencia a formar sus propios negocios al momento de su jubilación es mayor que en las personas jubiladas; centrando su actitud emprendedora en razones como: ya tienen un negocio familiar; desean trabajar de manera independiente; tienen experiencia en manejo de negocio, pero consideran que necesitan capacitarse más. Estas actitudes se encuentran casi en el 99\% de la masa crítica de los trabajadores que en los próximos cinco años se jubilarán.

85\% de las personas jubiladas y próximas a jubilarse consideraron que si ellos trabajan su dinero en un negocio propio, producirían

\footnotetext{
${ }^{8}$ Estudio cuantitativo mediante usos de muestras dirigido por el responsable de la investigación y desarrollado en el trabajo de campo mediante el apoyo de los alumnos del quinto ciclo de la Escuela de Administración de la Facultad de Ciencias administrativas de la Universidad Nacional Mayor de San Marcos durante los meses de Abril - Julio del 2016. El estudio se aplicó a personas jubiladas, por jubilarse, amas de casa y empresarios.
}

mayor cantidad de beneficio por su capital; en comparación de lo que le producen sus ahorros en una AFP.

El $71 \%$ de los jubilados manifestaron su deseo de capacitarse en La Universidad Nacional Mayor de San Marcos, si esta les ofrece algún programa o cursos de capacitación práctica; mientras que el $88 \%$ de los trabajadores próximos a jubilarse manifestaron la misma actitud.

En lo referente a los estudios realizados sobre situaciones, condiciones y percepciones en el mercado de amas de casa y empresarios, referentes sobre su disponibilidad de aceptación de los bienes y servicios ofrecidos por empresas formadas y gestionadas por personas jubiladas, el estudio descubre un escenario muy favorable en cuanto a sus percepciones y disposiciones de demanda.

Si bien es cierto que no ha sido una práctica de difusión de esta característica particular de la empresa, por la cual se ponga en relieve o de manifiesto que, existen empresas formadas y gestionadas por personas jubiladas desde hace tiempo atrás; en virtud de que por ley, cualquier ciudadano mayor de edad que no tenga ninguna restricción por ley, se encuentra facultado a formar una empresa. Es esta la razón que predomina en lo referente a que, en el pasado, esta razón no se constituía en algo relevante y es justamente la razón que hace que muchas empresas que tienen esa singularidad, reiteramos, no hayan considerado como aspecto relevante el destacar esta particularidad y, por la misma razón, muy pocas personas conozcan empresas formadas y gestionadas por jubilados. Empero, hoy las condiciones y circunstancias han cambiado; tal como se ha manifestado en la investigación. Hoy las expectativas de vida se han incrementado hasta los 75 años. El Sistema Privado de Pensiones no ha logrado cumplir con lo ofrecido a sus afiliados; la devolución de los ahorros de los trabajadores son devueltos por condicionamiento de la ley, en montos miserables y de hambre para el jubilado. El Perú es un país con altísima informalidad laboral ${ }^{9}$ (más del $75 \%$ de PEA se encuentra trabajando bajo condiciones de informalidad). El Estado continúa siendo el principal actor en el país

${ }^{9}$ Johannes Koettl (2005). Protección y Prácticas laborales del Banco Mundial - Caso España. 
que incumple las leyes laborales y maneja a su abusivo antojo el sometimiento de los trabajadores del país. Estas y otras condiciones laborales actuales hacen que el ciudadano, que llega a la edad de su Jubilación, sienta que debe continuar trabajando y produciendo ingresos; antes que someterse a vivir como ciudadano vulnerable, considerado así, la población jubilada.

$94 \%$ de las amas de casa de Lima no conocen empresas formadas por jubilados. Las pocas empresas conocidas trabajan en los rubros de cevicherias, servicios de seguridad, lavado de automóviles, servicios de construcción, bodegas, ferreterías, reparación de celulares y computadoras.

90\% de las amas de casa no tendrían ningún reparo en adquirir productos o servicios de empresas gestionadas por jubilados. $8 \%$ de las amas de casa desconfiarían de la calidad de los productos y servicios que pudieran ofrecer los jubilados y un $2 \%$ considerando que los jubilados son personas que están desactualizados.

$100 \%$ de las amas de casa estuvieron de acuerdo en que los jubilados trabajen su propio dinero. Este alto porcentaje en las amas de casa ha cambiado en el tiempo en razón de que, justamente, eran las esposas las que desconfiaban sobre los buenos resultados que pudieran obtener sus esposos en sus negocios. Este hecho constituye un factor favorable para el emprendimiento de los jubilados.

63 \% de las amas de casa manifestaron estar dispuestas ayudar a sus esposos en sus iniciativas emprendedoras. 38\% consideraron que sería una fuente de ingreso más para la familia, que se traduciría en mayor bienestar económico.

Otro importante componente de mercado fue la actitud y opinión de los micros y pequeños empresarios, referentes a las posibilidades comerciales que ellos podrían desarrollar con empresas de jubilados. 88\% de los empresarios manifestaron no tendrían ningún reparo en trabajar y adquirir los productos o servicios que estos pudieran ofrecer. $54 \%$ refirieron que el mayor cuidado que deben tener estas empresas estaría en las mismas condiciones que se exigen a cualquier empresa: buena calidad en sus productos y servicios.

Como se puede apreciar, en estos resultados de la investigación, la predisposición, expectativas y percepciones de las amas de casa y de los micros y pequeños empresarios se favorables para las empresas que se formen $\mathrm{y}$ gestiones por personas jubiladas y que será conveniente destacar en los procesos de comunicación de la empresa que estos formen.

\section{DISCUSIÓN}

La actitud de los jubilados actuales con respecto a su continuidad en la producción laboral ha cambiado diametralmente. El jubilado en nuestro país, hasta la década de los noventa, era una persona que aceptaba su cansancio a sus sesenta años; atribuyendo dicho estado a su "ancianidad", razón por la que, en acatamiento a las leyes laborales en cuanto a su retiro, aceptaba que la hora de su descanso laboral permanente había llegado y que, por lo tanto, debía retirarse a su hogar y vivir de una pensión que el Sistema Previsional le alcanzaría para su supervivencia. Asimismo, las esposas de los trabajadores se avenían a lo que buenamente el esposo jubilado recibía como pensión del Estado para vivir durante sus años de jubilación, una vejez "digna."

El avance en la medicina, en estos últimos treinta años ha sido de tal magnitud que las expectativas de vida se han incrementado en un tiempo considerable hasta los 75 años en países como el nuestro y en otros más desarrollados hasta los 80 años, como pronóstico de vida. Estos hechos, entre otros de orden financiero para favorecer al Sistema Privado de Pensiones a efectos que los ahorristas aporten por mayor cantidad de tiempo y dispongan de menor cantidad de plazo para la devolución de sus ahorros, ayudaría a las AFP a mejorar los montos paupérrimos de las devoluciones mensuales a sus ahorristas; razón que ha influenciado en la extensión de la edad de jubilación del trabajador, siendo hoy de 65 años para los varones y de 60 años para las damas.

Actualmente la mentalidad de las esposas y los propios jubilados acatan la edad de sus jubilaciones, empero siente que aún poseen las fuerzas físicas y sus voluntades para 
continuar laborando y producir ingresos. Altos porcentajes de ellos siente y piensan que es sumamente importante capacitarse para emprender un negocio; lo cual unido a su experiencia laboral y algún capital, siente que son capaces de producir mayor renta que la que le ofrecen las AFP por sus ahorros. Sienten que tienen muchos años por delante para producir $\mathrm{y}$ tener mejores niveles de vida conjuntamente con sus familias. Y alcanzan a comprender que el Estado se ha permitido que sean ellos mismos los que tengan que velar por su seguridad y supervivencia.

En nuestro país, el sistema previsional conformado por el sistema estatal y el Sistema Privado de Pensiones, AFP, en la práctica, han demostrado ser sistemas generadores de grupos vulnerables; que condenan a la pobreza al ciudadano trabajador, después que este ha entregado a la sociedad los mejores y mayores años de su mayor fortaleza y productividad. La jubilación para el trabajador es un verdadero shock psicológico y, al mismo tiempo, un shock económico en virtud a que sus ingresos caen en más del $65 \%$, en relación a sus últimas remuneraciones.

En países de mayor desarrollo, esta población de trabajadores que se jubilan y jubilarán o son dejados al abandono ni a su suerte: existen programas de preparación psicológica para la mejor salida y adaptación del jubilado a su nueva vida outplacement y, de igual forma, existen programas de motivación para el emprendimiento para quienes deseen iniciar algún tipo de negocio, asociarse con otros jubilados o personas interesadas en el negocio propuesto. La investigación demuestra la inexistencia de estos mecanismos en nuestras leyes laborales y sistema laboral. Con la existencia de un programa de preparación y capacitación de trabajadores que se jubilarán, se obtendrá muchísimos beneficios sociales en estos grupos y se evitaría, en gran medida, que los jubilados constituyan un grupo vulnerable en nuestra sociedad.

\section{CONCLUSIONES}

En nuestro país se dan leyes celestiales, maravillosas, geniales y envidiables para otros países tal vez; empero, la realidad es que estas leyes solo están en el papel. Las leyes no se cumplen por falta de implementación, reglamentación; empero, fundamentalmente por falta de presupuesto, infraestructura y medios para sus fines. La ausencia de presencia del Estado en infinidad de áreas, campos y niveles de la vida e interacción del ciudadano y población el estado siempre está ausente.

La jubilación se entiende en nuestro país como un sinónimo de vejez de la persona; esto ya no es así en muchos países del mundo, en razón de que la ciencia médica ha logrado incrementar el horizonte de esperanza de vida de las personas, siendo esta hoy en nuestro país, una media de 74 años. Es por este hecho que algunos denominan el inicio de la cuarta edad, al trabajador que se jubila por ley a los 65 años, tiene y debe continuar trabajando hoy a efecto de hacerle frente a la caída vertiginosa de sus ingresos, situación que lo convierte en población vulnerable y definitivamente lo empobrece en razón de que, su poder adquisitivo cae fuertemente en un rango entre 60 a $65 \%$ en relación a sus últimos sueldos, esto solo tiene un nombre: empobrecimiento. Lo cual se debe sumar a la pobreza del Estado con su pésima atención en salud para el jubilado y/o adulto mayor.

En nuestro país no existe un programa oficial público y privado de preparación y capacitación del trabajador próximo a su salida del mercado laboral, en términos de su jubilación que lo considere y proteja. Los trabajadores del país se encuentran desamparados a su suerte una vez que se jubila y enfrente el shock psicológico del retiro laboral inconsulto y obligatorio; aun cuando él se siente en plenas facultades psicológicas y físicas para continuar produciendo y procurándose ingresos decentes para sí y su familia.

Existen condiciones sociales y de cambio de percepciones que se tornan apropiadas tanto desde la población de adultos mayores jubilados y por jubilarse, como también por el entorno político consciente de avanzada para cambiar esta situación de desamparo que deben enfrentar los trabajadores del país. Hoy los trabajadores adultos saben que tras su jubilación y dotados de sus buenas facultades psíquicas y físicas pueden proyectar su vida generando ingresos hasta los 75 años y escapar 
de la pobreza y miseria a la que son condenados por ambos sistemas previsionales en nuestro país.

Las bajas pensiones, que brindan los sistemas previsionales, no son responsabilidad de los trabajadores sino de la mala administración en general que ha tenido el país en las últimas tres décadas. Una de las causas más fatales es la variabilidad de regímenes laborales existente en el sector público y en el privado; otra es el fomento desde el Estado con leyes que los empresarios ni los trabajadores aceptan y que, por consecuencia, generan informalidad laboral muy alta. Otro aspecto que impacta fuertemente en las magras remuneraciones de los jubilados es el bajo nivel remunerativo del sueldo mínimo vital. El cuarto factor de mayor relevancia en el fracaso en las bajas "pensiones" de los jubilados ha sido la creación del Sistema Privado de Pensiones, siendo este un sistema a todas luces una estafa para el trabajador y su familia y una enorme fuente de riqueza para los inversionistas en las administradoras monopólicas de los Fondos de Pensiones y su socio el Estado; que se sirve de ellas financiándose con recursos económicos confiscados del sueldo de los trabajadores y que llenan sus arcas con recursos económicos provenientes del sudor del pueblo.

Los jubilados del Perú no pueden continuar desprotegidos, viviendo con miserables devoluciones de sus ahorros bajo denominación de "Pensión mensual"; termino mentiroso y estafador que sume a los jubilados en largos años de pobreza y miseria.

Otro aspecto relevante que debemos señalar en esta investigación es que en la revisión de diversa literatura y estadísticas, se ha podido evidenciar que el Estado es el primero en incumplir las leyes y obligaciones con sus trabajadores y con los pagos que debe hacerse al sistema previsional tanto público como privado. Tampoco, el Estado cumple con pagar y depositar la CTS (compensación por tiempo de servicio) de los trabajadores. El hito histórico en la administración del Estado, sobre este particular, se da en la sinvergüencería de la única indemnización pagada al asesor del Presidencial Vladimiro Montesino por su tiempo de servicio de S/. 15’000,000.00 mientras los trabajadores jamás recibieron un solo centavo.

Los jubilados actuales han cambiado radicalmente su actitud y comprenden que luego de su retiro del mercado laboral aún cuentan con suficientes fuerzas y salud para salir de la pobreza a la que lo condena su estado. Y, para liberarse de ello, deben continuar laborando con sus experiencias y pequeños capitales producto de la devolución de sus ahorros por las AFP.

Altísimo porcentaje de jubilados y adultos mayores están dispuestos a capacitarse en Universidad Nacional Mayor de San Marcos para conducir eficientemente sus emprendimientos y procurarse mayores ingresos trabajando su dinero y el de su familia, mediante el emprendimiento.

\section{REFERENCIA BIBLIOGRAFICA.}

Acta bioeth. v.7 n.1 Santiago 2001. http://dx.doi. org/10.4067/S1726-569X2001000100008

Acs, Z. J., \& Amorós, J. E. (2008). Introduction: Thestartupprocess. Estudios de Economía, 35(2), 121- 132.

Andrew Shepherd (2004) Una mirada a la protección social desde los derechos humanos y otros contextos internacionales. Publicación de las Naciones Unidas ISSN impreso 1564-4162 ISSN electrónico 1680-8983.

Barraza de Anda M. La Productividad Social en las universidades Públicas en México- XI Congreso Internacional sobre la Reforma del Estado y la Administración Pública

Castells, M. (2000). "Era de la información, la economía, sociedad y cultura: la sociedad red". Volumen 1. Siglo veintiuno Editores. 580 páginas.

Centro de Innovación de la Universidad de Chile (2014-2) Los emprendimientos por necesidad a los emprendimientos por oportunidad. Denise Díaz.- Cristhian A. Cansino

Davenport, T. (1999). “Ecología de la Información. Porqué la tecnología no es suficiente para lograr el éxito en la era de la información". Oxford UniversityPress. USA. 296 Páginas

Estudio cuantitativo mediante usos de muestras dirigido y aplicado por el responsable de la investigación y desarrollado en el trabajo de campo mediante el apoyo de los alumnos del quinto ciclo de la Escuela de Administración de la Facultad de Ciencias administrativas de 
la Universidad Nacional Mayor de San Marcos durante los meses de Abril - Julio del 2016. El estudio se aplicó a personas jubiladas, por jubilarse, amas de casa y empresarios.

Johannes Koettl (2005). Protección y Prácticas laborales del Banco Mundial - Caso España.

Lehr y Pholman, 2000 Participación de las personas mayores en el mercado de trabajo y en la sociedad. Revista Española de Geriatría y Gerontología, 39, 180-84.

Loretto, W. (2010). Work and retirement in an ageing world: the case of olderworkers in the UK. Twenty-First Century Society, 5(3), 279294. http://dx.doi.org/10.1080/17450144.201 0.480831

Reynolds, W. D. (2002). Global Entrepreneurship Monitor: 2002 executivereport. Londres: Babson.

Recomendación sobre los trabajadores de edad, 1980 (núm. 162) 23 de junio de 1980 Ginebra - Suiza
Sandra Torrades Bióloga (2002). Farmacogenética: la medicina a la carta. Vol 21 Núm 10 Noviembre 2002 Pág. 126 - 130

Segunda Asamblea Mundial sobre el Envejecimiento (8-12 de abril 2002 - Madrid, España)

Texto Unico Ordenado del Dec. Leg. 728, ley de productividad y competitividad laboral decreto supremo 003-97-tr 27/03/1997

OECD-2012 PolicyBriefon Senior Entrepreneurship. Entrepreneurial Activities in Europe Retrieved from http://ec.europa.eu/social/ BlobServlet?docId=9644\&langId=en

Oms (Organización Mundial de la Salud) (2012). La buena salud añade vida a los años Retrieved from:

1. h t t p : // a p p s.who.int/i r i s / bitstream/10665/75254/1/WHO_DCO_ WHD_2012.2_spa.pdf

2. Víctor Ruíz (2009) - FORUM LIBERTADES. COM - Diario digital 